\title{
Contribución de las mujeres en las revistas de sociología colombianas, 1959-2000
}

Contribution of women in Colombian sociology journals, 1959-2000

Mayra Alejandra García Jurado ${ }^{1}$

Universidad Santo Tomás (Colombia)

Artículo de investigación

Fecha de recepción: Marzo 23 de 2018

Fecha de aceptación: Agosto 30 de 2018

\section{Para citar este artículo}

García, M. A. (2018). Contribución de las mujeres en las revistas de sociología colombianas, 1959-2000. Revista Campos en Ciencias Sociales, 6(1), 7390. Bogotá: Universidad Santo Tomás. DOI: https://doi.org/10.15332/ s2339-3688.2018.0001.03

1 Socióloga. Correo electrónico: mayragarcia@usantotomas.edu.co 


\section{RESUMEN}

La contribución de las mujeres en la producción de conocimiento científico ha sido un fenómeno poco reconocido en la comunidad académica, en el caso de la sociología se menciona de forma constante el trabajo realizado por los "padres fundadores", y poco se habla del desarrollo del conocimiento sociológico realizado por mujeres, a pesar de que sus contribuciones se evidencian desde el siglo XIX; se pretende con el presente estudio realizar un análisis documental y bibliométrico de la producción científica de las mujeres sociólogas colombianas entre los años 1959 y 2000 , tomando como referencia las dos únicas instituciones nacionales (Universidad Santo Tomás y Universidad Nacional de Colombia) que realizaron publicaciones y permanecieron activas en dicho periodo. Se encuentra que existe poca representación de las mujeres en la producción sociológica, sin embargo, estuvieron activas en la totalidad del periodo de tiempo delimitado, siendo su mayor contribución el desarrollo conceptual de la disciplina.

Palabras clave: mujeres, sociología, bibliometría, contribución.

\section{Abstract}

The contribution of women in the production of scientific knowledge has been a phenomenon not very recognized in the academic community, in the case of sociology is constantly mentioned the work done by the "founding fathers" and little is said about the development of Sociological knowledge carried out by women, despite the fact that their contributions have been evident since the 19.th century. With this study, it is intended to carry out a documentary and bibliometric analysis of the scientific production of Colombian sociologist women between 1959 and 2000, taking as reference the only two national institutions (Universidad Santo Tomás and Universidad Nacional de Colombia) that published and remained active in that period. It is found that there is little representation of women in the sociological production, despite the fact they were active in the totality of the delimited period of time, being their main contribution the conceptual development of the discipline.

Keywords: Women, sociology, bibliometrics, contribution. 
Mayra Alejandra García Jurado

\section{INTRODUCCIÓN}

El presente artículo se basa en una tesis personal para optar por el título de socióloga en la Universidad Santo Tomás, titulada "La inserción de la mujer en la sociología 1959-1960 en Bogotá D. C.”, desarrollada a lo largo del año 2017. La necesidad de realizar una investigación que logre sistematizar la participación de las mujeres en el desarrollo del conocimiento sociológico en Colombia, se origina a partir de la preocupación al encontrar pocos estudios que hagan referencia a este tema en el país. A pesar de que existen diversas luchas materializadas en investigaciones que han revindicado el papel de las mujeres en la sociología, como las diferentes obras desarrolladas por Magdalena León y Luz Gabriela Arango, es urgente comprender de manera general y legítima la participación de la mujer en esta disciplina.

Según la comunidad académica, la sociología fue desarrollada en Europa a mediados del siglo XIX, y su estructura conceptual fue constituida por hombres como Auguste Comte, Herbert Spencer, Émile Durkheim, Max Weber, Karl Marx y Talcott Parsons, quienes se conocen como los "padres fundadores". La afirmación de que estos personajes fueron los grandes pensadores de la disciplina, ha dejado de lado la contribución de las mujeres en la sociología, desconociendo por ejemplo los trabajos realizados por Marianne Weber y Betraice Webb a finales del siglo XIX. A pesar de que se han llevado a cabo esfuerzos para lograr este reconocimiento, "la comunidad académica no las reconoce como miembros productivos, y lo anterior puede evidenciarse en los diferentes espacios académicos que se generan en sociología (planes de estudio, eventos académicos, producción de investigaciones, reconocimientos, entre otros) (García, 2017, p. 18).

La situación a nivel local no fue diferente, en 1959 la sociología se posiciona como profesión con las primeras facultades en Bogotá, inicialmente con el surgimiento del programa en la Universidad Nacional de Colombia, luego en la Pontificia Universidad Javeriana y en la Universidad Santo Tomás. Así como la sociología general, en Colombia Orlando Fals Borda y Camilo Torres, junto con mujeres como María Cristina Salazar y Virginia Gutiérrez hicieron parte del proceso institucionalización de la sociología en el país; y pese a que las mujeres hicieron un trabajo paralelo con los hombres, es poco o casi nulo el reconocimiento de la producción femenina en la comunidad académica. 
Entendiendo este fenómeno en la sociología colombiana, surge la necesidad de visibilizar y analizar la contribución de las mujeres en la producción académica, este tipo de análisis permite, además, comprender las situaciones de representación y reconocimiento de las mujeres en el escenario académico, y es por ello que se recurre a la sociología de la ciencia, perspectiva estructural funcionalista que conceptualiza la organización y normatividad de las comunidades científicas a partir de la premisa que afirma que la ciencia es el conocimiento más organizado y legitimado por la sociedad, pero además dando cuenta de las influencias sociales, históricas y culturales que se encuentran detrás de la producción científica.

Siguiendo la anterior perspectiva y para el cumplimiento del objetivo de esta investigación, se realiza un análisis bibliométrico de las producciones académicas seriadas que se desarrollaron en la época en dos universidades: Universidad Nacional de Colombia y Universidad Santo Tomás. Aunque esta técnica se utiliza principalmente para medir el impacto de la producción científica o para realizar una descripción de esta, en el presente caso las observaciones se centran en indicadores de género, lo que finalmente permite visibilizar dicha contribución y analizar la representación femenina dentro de la comunidad académica de la época.

\section{El efecto Matilda y los fenómenos de segregación femenina en la ciencia}

Es bien sabido que la representación de las mujeres en la ciencia y puntualmente en la sociología es insuficiente, lo anterior no se debe a una -escasa- contribución femenina al conocimiento, sino a un fenómeno social de segregación presente en las dinámicas de la comunidad científica-académica. Harriet Zuckerman y Robert Merton (1977) identificaron y conceptualizaron las dinámicas generales de las comunidades científicas, dentro de las cuales se encuentra el sistema de recompensas que funciona a partir de la estratificación de recompensas ${ }^{2}$ a los productos científicos, los cuales a su vez se califican por medio de un juicio de pares que -según Merton (1977) - tiene como criterio unos valores institucionalizados (ethos) en los cuáles prima la aplicación de normas técnicas, teóricas y metodológicas, la difusión del

2 Estas recompensas pueden entenderse como valoración, legitimación y difusión del producto científicoacadémico y por lo tanto reconocimiento a quien realizó el producto. 
conocimiento y la evaluación por méritos, dejando de lado las características sociales de los autores.

Es claro que esta conceptualización no obedece a una realidad neutral de la valoración de los productos científicos, sino a un deber ser de la misma y es por esta razón que los autores identifican en el sistema de recompensas el efecto Mateo, que ocurre cuando a pesar de que un producto científico de gran impacto es desarrollado de forma colaborativa, quien se lleva el reconocimiento por dicha contribución es una sola persona, la cual previamente fue reconocida en el círculo, a pesar de que los demás miembros del equipo (los demás autores) hayan aportado de forma significativa a esa contribución.

Este efecto influye tanto al sistema de recompensas (al no ser neutral en la valoración de los productos científicos) como al sistema de comunicación, es decir, afecta también la difusión y divulgación de tales productos. Pero además, el efecto Mateo puede evidenciarse de manera particular en el caso de las mujeres. Rossiter (1993) reconceptualiza este efecto teniendo en cuenta las repercusiones sistemáticas del mismo hacia las mujeres y lo traduce en el efecto Matilda, argumentando que la imparcialidad de la evaluación y legitimación de los productos científicos aumenta en el caso de las mujeres, resultando en repercusiones que se pueden ejemplificar en cientos de casos.

Puede mencionarse entre ellos el nombre de una de las primeras matemáticas afroamericanas en culminar un posgrado (1938): Katherine Coleman Globe Johnson, quien con el apoyo de otras dos mujeres solucionó y calculó el regreso de la misión de la NASA Apolo 13 - una de las más importantes en la década de los 60-, sin embargo, este logro le fue reconocido casi 20 años después por medio de una premiación; así, también existen casos en la sociología, véase por ejemplo el caso del primer programa de sociología que se fundó en la Escuela de Chicago en 1895, en el cual se aceptó con normalidad el ingreso de mujeres a sus aulas, hasta que en $1902 \mathrm{el}$ entonces decano William Rainey decide abrir un College Junior para mujeres, donde solo se abordaban temáticas relacionadas con trabajo social, infancia, estadística y ciencias sanitarias (García, 2010), pese a que varias estudiantes de la época, como Jane Addams, aportaron en temas de guerra, democracia, política y crítica social. 
El efecto Matilda explica el fenómeno del poco reconocimiento y representación de las mujeres en la sociología, a partir de las dinámicas sociales presentes en las comunidades científicas-académicas que finalmente han sido permeadas por el contexto social e histórico de segregación femenina a nivel global. Este contexto trae consigo barreras sociales que han dificultado dicho reconocimiento, pero ello se puede contrarrestar con el rescate y visibilización de los aportes a la disciplina realizados por mujeres, en este caso a nivel nacional como punto de partida.

\section{Revistas y difusión del conocimiento en Colombia}

Como un ejercicio de reconocimiento y visibilización a los aportes de las mujeres sociólogas en Colombia, se realiza un análisis de documentos académicos, en este caso de revistas o publicaciones seriadas difundidas entre el año 1959 y el año 2000; se hace énfasis en estas publicaciones, ya que en su momento, y aún hoy en día, son la materialización de las investigaciones realizadas por parte de una persona, entidad, universidad o institución académica, es decir, los documentos académicos son fundamentales en las diferentes etapas de la actividad investigativa y además evidencian el progreso científico (Urbizagástegui y Restrepo, s.f.).

En el país las revistas científicas y académicas han sufrido grandes cambios a partir de 1990 con la implementación de Publindex como herramienta institucional de clasificación de las revistas nacionales. Antes de esa década la producción, edición y distribución de estas funcionaba de forma peculiar, puesto que no existía reglamentación de producción científica en su edición y homogenización, esto quiere decir que no eran consecutivas y su contenido no estaba limitado a artículos de investigación.

Lo anterior puede evidenciarse en diversas revistas, en donde hubo periodos de nula producción y el grosor de algunos números en los que se acumulaba la labor investigativa por dos años de una facultad. Estos desfases editoriales fueron efectos de la informalidad con la que se producían las revistas, aclarando que por lo general, las revistas antes de los 90 se desarrollaban gracias a iniciativas de docentes o administrativos de una institución académica, generalmente con el deseo de difundir los resultados de investigaciones personales o institucionales, y por lo mismo eran 
estos personajes quienes se encargaban de la edición de las revistas, fuese por voluntad o carga administrativa (Suárez, 2017).

Sin embargo, la informalidad de la producción de revistas no significaba poca o baja calidad de las investigaciones que se realizaron, por el contrario, las revistas contenían los temas y las problemáticas que más se trataron en la época.

\section{Metodología}

El marco metodológico que guió la toma y el análisis de datos de esta investigación obedece al paradigma empírico analítico, cuestión que indica la utilización de una metodología cuantitativa, en este caso el análisis de los datos se hizo a partir de la técnica de bibliometría, desde la cual se analizó la contribución de las mujeres en las revistas colombianas de sociología, a través de indicadores que permitieron desagregar los artículos elaborados por estas.

La bibliometría es una técnica que mide la actividad científica con la utilización de indicadores que generalmente se basan en las publicaciones y en las citas para conocer el impacto académico y desarrollo de documentos publicados por una institución, país o individuo (Licea y Santillán, 2002). Esta herramienta se popularizó a finales del siglo XX, gracias al surgimiento de las bases de datos electrónicas, factor que facilitó la recolecta y análisis de los datos bibliográficos.

En Colombia se encuentran algunos estudios sobre producción científica local, desarrollados muchas veces por la conmemoración de una revista con un largo recorrido o la fundación de una facultad o departamento universitario; estos estudios se centran, por lo general, en el análisis descriptivo de la producción académica de una institución o disciplina a nivel local. Además, cabe mencionar la existencia de estudios de análisis de la producción académica enfocados en el contexto social (Jaraba, Guerrero, Gómez y López, 2011).

Para este caso en específico, se hace uso de la bibliometría para comprender la contribución de las mujeres en las revistas de sociología colombianas, visibilizando los artículos en donde se materializaron los resultados de sus investigaciones. 
En una primera fase, se realizó la recolección de datos pertenecientes a tres fuentes; la primera obedece a un documento bibliográfico que contiene las publicaciones del Departamento de Sociología de la Universidad Nacional de Colombia entre los ańos 1959 y 1979 (Varón, 1979), es decir, las producciones académicas realizadas en el Departamento previas al lanzamiento de la Revista Colombiana de Sociologia; anterior a esta revista existieron publicaciones seriadas como Monografias Sociológicas, estos datos se obtuvieron de forma virtual desde el repositorio institucional que brinda la Universidad Nacional de Colombia. Esta fuente develó los primeros acercamientos de las mujeres al conocimiento sociológico, comprendiendo que estos documentos fueron legitimados por la comunidad académica del momento.

La segunda fuente se trata de la publicación seriada Cuadernos de Sociología vinculada con la Facultad de Sociología de la Universidad Santo Tomás, la cual inicia su difusión en el año de 1977, esta exploración se llevó a cabo en físico en la hemeroteca de la Biblioteca Fray Luis J. Torres O. P.

La tercera fuente corresponde a la Revista Colombiana de Sociología, que surgió en el año 1979, la revisión de esta revista se realizó por medio digital (https://revistas.unal. edu.co/index.php/RECS). Estas fuentes son de gran pertinencia, ya que fueron las únicas publicaciones de este tipo existentes en la época a nivel nacional.

Posteriormente, en una segunda fase se sistematizó la información a través de una base de datos que contiene las siguientes categorías : año de publicación, volumen y número, tipo de texto, tema del texto (a partir de categorías y subcategorías), desagregación por sexo, coautoría y total de artículos por volumen; esta categorización permitió el análisis efectivo de los datos, teniendo en cuenta que los indicadores que usualmente se utilizan para el análisis de las revistas académicas, acatan a objetivos de soporte y evaluación vigentes en las políticas públicas del sector de ciencia y tecnología (Daza y Pérez, 2008).

El ejercicio de "contar" la cantidad de artículos que publicaron las mujeres en revistas se aplica con el fin de referir de forma empírica la participación de estas en la sociología institucionalizada en estas universidades, teniendo en cuenta que en los diferentes documentos acerca de la historia de la sociología en Colombia, no se encuentra ningún apartado con indicadores que desagreguen por sexo. 


\section{Resultados}

A continuación, se presentan los resultados obtenidos y su análisis, en un primer momento se describe de forma breve las características de las tres fuentes analizadas, para luego exponer de forma aglomerada la contribución de las mujeres en estas revistas, se consideró la forma más efectiva para presentar los resultados teniendo en cuenta que aislar los datos de cada publicación periódica daría resultados poco comprensibles.

\section{Publicaciones seriadas del Departamento de Sociología de la Universidad Nacional de Colombia, 1959-1979}

Desde sus comienzos, los miembros de la Facultad y posteriormente el Departamento de Sociología de la Universidad Nacional de Colombia, se preocuparon por la difusión de la producción académica, esto se evidencia con su primera publicación seriada que puede considerarse la primera del país: Monografías Sociológicas, la cual durante los 7 años de su circulación (de 1959 a 1967), publico 14 números a nombre de diversos personajes, entre los que se encuentran Virginia Gutiérrez, Orlando Fals Borda y Camilo Torres, quienes se consideran "fundadores de la sociología en Colombia".

Luego de unos años de la desaparición de Monografias Sociológicas, surge en 1970 Cuadernos de Sociología, de la cual hacen parte 6 números publicados hasta el año 1973; en esta publicación aparecen personajes como Carmenza Gallo y Gabriel Restrepo, quienes representaron una nueva generación de sociólogos en la Universidad Nacional de Colombia. Posteriormente, en 1977, aparece la publicación Documentos de Sociología, serie que dejó de circular en 1979 y sin embargo publicó 19 números, contando con la participación de varios sociólogos pertenecientes a esa nueva generación, en la que se evidenció además la participación de mujeres como Anita Weiss.

Esta información evidencia los primeros rastros de aportes de las mujeres a la sociología, Virginia Gutiérrez y Anita Weiss, fueron pioneras en la publicación de productos académicos con estudios sobre la cultura popular colombiana y la participación política en el país. Aunque Virginia Gutiérrez de Pineda fue formada en antropología, es indiscutible que fue una de las primeras mujeres 
en ser parte de la comunidad académica de la sociología en Bogotá, e inspiró una línea que pronto estaría en auge en las investigaciones sociológicas del país: la familia. (García, 2017, p. 44)

Se revisaron en total 69 artículos que se pueden considerar como una de las producciones más influenciables en la sociología colombiana de la época, y de su distribución pueden concluirse algunos fenómenos sociales en la comunidad académica.

\section{Cuadernos de Sociología, Universidad Santo Tomás}

En el año de 1977 surge en la Facultad de Sociología de la Universidad Santo Tomás la publicación seriada Cuadernos de Sociología, se concentra en ser una herramienta para estudiantes y docentes interesados en las ciencias sociales, y como objeto de la mayoría de las revistas científicas, se enfocó en la difusión académica producida por el Centro de Investigación de la Facultad, por estudiantes y docentes (Páez, 1977).

De esta publicación se analizaron 23 años de producción, de 1977 hasta el 2000, se evidencia una gran variación en el número de artículos publicados por año, lo que significa un esfuerzo por mantener vigente la serie. De 1996 a 1998 fue nula la producción, probablemente por cambios administrativos que intervinieron en la edición y estructura de la revista. Esta publicación seriada fue la más efectiva de la época, publicando 111 artículos en 20 volúmenes.

En cuando a la participación de mujeres en esta publicación, se encuentra un 16 $\%$ frente al $84 \%$ de artículos publicados por hombres, teniendo en cuenta que durante varios años la editora fue Alina López de Rey. Por otro lado, se evidencia que la mayoría de mujeres que allí publicaron fueron parte de la planta docente de la Facultad, plasmando allí los resultados de sus investigaciones, como el caso de Ana Dolores Medina de Ruiz y María Victoria C. de Fornier, quienes trabajaron temas de estratificación social, familia y aportes de la sociología al desarrollo del país. 


\section{Revista Colombiana de Sociología, Universidad Nacional de Colombia}

La Revista Colombiana de Sociología ha sido la serie de publicaciones más duradera del Departamento de Sociología de la Universidad Nacional de Colombia. Tuvo como objetivo convertirse en "un espacio para la reflexión y análisis de lo social en una perspectiva científica, nacional y política” (Restrepo, 1979, p. 3), lanzando su primer número en el año 1979 y hasta el año 2000 publicó 118 artículos en 15 volúmenes, a pesar de suspender intermitentemente su difusión por 9 años durante este periodo de tiempo. Se evidencia también que del $100 \%$ de los artículos publicados durante este periodo, el $9 \%$ fueron desarrollados por mujeres.

La participación femenina estuvo representada por mujeres como Luz Teresa Gómez de Mantilla, Diana Obregón Torres, Anita Weiss, Luz Gabriela Arango y Olga Restrepo, quienes trataron temas de conceptualización sociológica, métodos de investigación y género.

Finalmente, se exponen los resultados generales de los datos descritos anteriormente, teniendo presente que aislar el análisis de cada publicación seriada no sería suficiente para evidenciar la contribución de las mujeres en las revistas de sociología colombianas del año 1959 al 2000, en vista del pequeño porcentaje de participación en las revistas; sin embargo esta cantidad no es un indicador de la contribución a la disciplina, dado que, como lo señala Magdalena Velázquez:

La tajante división entre las actividades masculinas y femeninas, los criterios de valoración social de unas y otras y la asignación exclusiva al varón del ejercicio del poder en la vida social, económica, política y familiar, han conducido a que el que hacer de las mujeres sea una presencia ausente en nuestro discurrir histórico. La ausencia de las mujeres de la vida pública las ha hecho también invisibles para la historia, pero allí, desde esa carencia, las mujeres han tenido un desempeńo específico. (Velásquez, 1989, p. 10)

De modo que es posible concebir como grandes contribuciones los artículos publicados por estas mujeres, teniendo en cuenta que a pesar de un contexto complejo y lleno de factores adversos para su desarrollo y reconocimiento científico, algunas de ellas se encontraron como autoras en la época, y estas pueden percibirse como representantes de aquellas que a pesar de seguir las normas científicas e institucionales 
de la comunidad académica, fueron invisibilidades por barreras sociales, políticas y culturales.

Tabla 1. Porcentaje de firmas en publicaciones seriadas 1959-2000

\begin{tabular}{|c|l|c|c|c|}
\hline Año & Publicación seriada & \% Firmas FEM & \% Firmas MAS & \% Coautoría \\
\hline $1959-1979$ & $\begin{array}{l}\text { Otras publicaciones dep. } \\
\text { Sociología UN }\end{array}$ & $12 \%$ & $65 \%$ & $23 \%$ \\
\hline $1977-2000$ & Cuadernos de Sociología & $21 \%$ & $59 \%$ & $20 \%$ \\
\hline $1979-2000$ & $\begin{array}{l}\text { Revista Colombiana de } \\
\text { Sociología }\end{array}$ & $10 \%$ & $87 \%$ & $3 \%$ \\
\hline
\end{tabular}

Fuente. Elaboración propia

Como se observa en la tabla 1, la distribución de firmas masculinas, femeninas y de coautoría es muy variable entre publicaciones, se evidencia que en general el porcentaje de firmas de mujeres es siempre menor, con un máximo del $21 \%$ en la revista Cuadernos de Sociología, y un mínimo de un 10 \% en la Revista Colombiana de Sociología, que el fenómeno de poca representación femenina se haya generalizado por casi 40 años puede significar la existencia de barreras sociales que no permitieron la participación de las mujeres de manera efectiva en las publicaciones seriadas, lo anterior se sustenta por la permanencia de mujeres como Anita Weiss en la planta docente.

Teniendo en cuenta que en su momento estas publicaciones seriadas eran editadas y producidas por la misma planta docente de cada universidad, se encontró un patrón de firmas recurrentes en el caso de las publicaciones del Departamento de Sociología entre 1959 y 1979 de hombres como Orlando Fals Borda y Eugene Havens, mientras que mujeres como Virginia Gutiérrez tienen máximo 2 firmas. Lo que evidencia que, a pesar de su participación en la institucionalización de la sociología del país, el fenómeno en el sistema de recompensas -efecto Matilda- dificultó la participación de mujeres en las publicaciones seriadas. Pese al cambio editorial y reglamentación de las revistas académicas esta situación se sigue presentando en la actualidad en diferentes disciplinas, el estudio realizado por Moss-Racusin, Dovidio, Brescoll, Graham y Handelsman (2012) demuestra que, aunque el fenómeno se ha reducido, los hombres siguen dominando la producción científica en la mayoría de los países. 
En cuanto al porcentaje de coautoría, se evidencia que llega al máximo en las publicaciones del Departamento de Sociología de la Universidad Nacional de Colombia, entre 1959 y 1979 con un $23 \%$, una situación paradójica dadas las ventajas de la publicación en coautoría con la regulación de la producción científica en 1990. La situación se presentó en la revista de la Universidad Santo Tomás - Cuadernos de Sociología-, esto pudo haber ocurrido porque en la época la publicación de artículos tenía un valor simbólico de legitimidad, contrario a la situación actual, en la que publicar un artículo trae consigo beneficios laborales e inclusive salariales. Este resultado también puede ser efecto de las publicaciones realizadas en conjunto por la Asociación Colombiana de Sociología o por grupos de investigación pertenecientes a las universidades examinadas.

Ahora, para enfocar el análisis a la producción realizada por mujeres, se presenta la siguiente figura de distribución aglomerando las diferentes publicaciones seriadas:

Figura 1. Distribución de publicaciones realizadas por mujeres 1959-2000

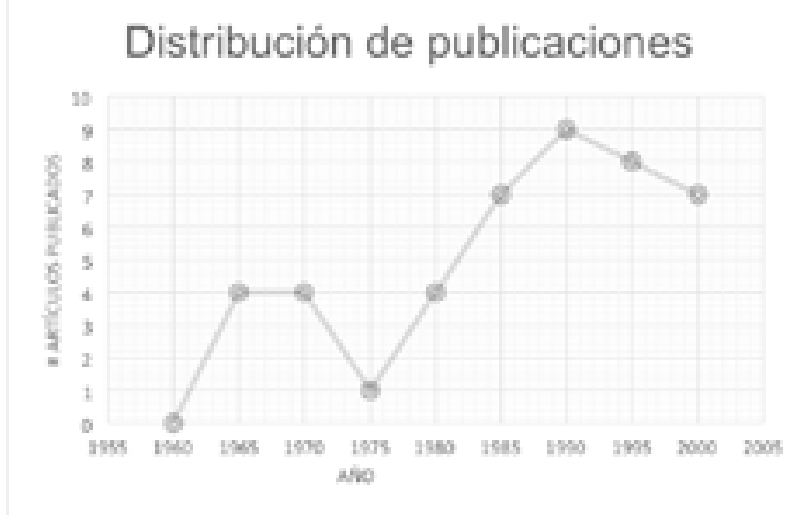

Fuente. Elaboración propia

En la figura 1 se evidencia el número de artículos publicados por mujeres desde al año 1959 hasta el año 2000, esta gráfica se realizó con la totalidad de artículos realizados por mujeres (44 artículos en total). Se observa que en las primeras décadas de las revistas hubo poca participación de mujeres, esto pudo haber sido gracias a diferentes factores como el reciente acceso legítimo de mujeres a la educación superior, o el poco porcentaje de mujeres en la planta docente y administrativa de las universidades 
en las que se encontraron vinculadas las revistas; el pico descendiente en el año de 1975 pudo ser producido por lo cierres temporales de la facultad o departamento de sociología en las universidades que ocurrieron por esa época, a partir de 1980 se evidencia una tendencia de aumento en el número de artículos publicados por mujeres hasta el pico más alto de participación en 1990 con 10 artículos publicados en ese año.

El aumento progresivo pudo ser fomentado por las nuevas generaciones de sociólogas que se formaron entre la década de los 70 y los 80 , quienes incursionaron en nuevos temas de carácter científico social y de pertinencia nacional como la sociología de la ciencia y el desarrollo económico e industrial. De cualquier manera, es preocupante dar cuenta que de 299 artículos revisados, solo 44 (el $14 \%$ ) fueron firmados por mujeres, yademás que aun esta cifra no fue suficiente para reconocer los aportes de estas al desarrollo de la disciplina en el país, esta falta de reconocimiento y representación sigue reproduciendo cifras similares hoy en día, "la escasa representación de las mujeres en la ciencia hasta el momento ha provocado que los hombres progresen en sus carreras científicas hasta ocupar la mayoría de los altos cargos, esto podría explicarse como consecuencia de las fuerzas históricas” (Bea, 2016, p. 10).

Figura 2. Temáticas desarrolladas por mujeres en las publicaciones académicas en sociología $1959-2000$

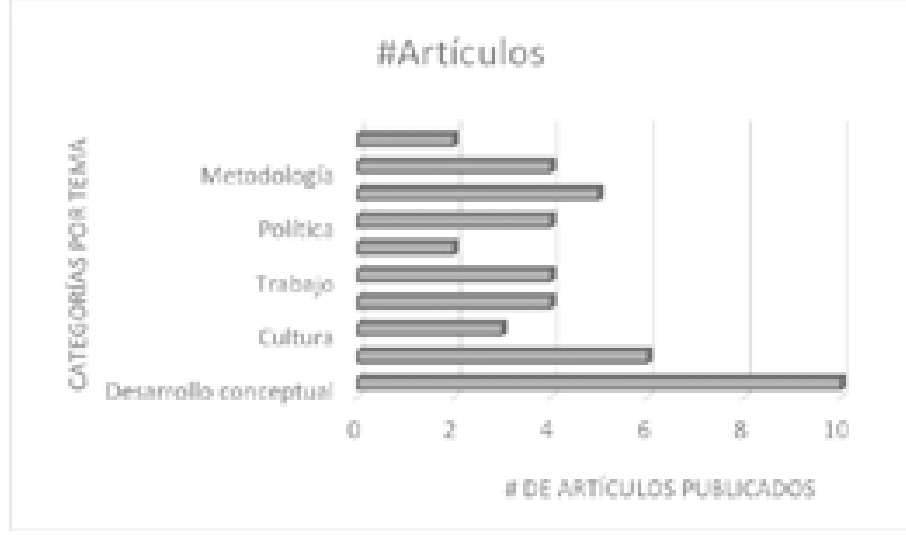

Fuente. Elaboración propia 
En la figura 2 se evidencian las temáticas desarrolladas por mujeres en las revistas colombianas de sociología, se exponen allí los ejes temáticos dentro de los cuales se inscribieron los artículos, en términos generales, existió un equilibrio en los temas que trabajaron las mujeres, como lo son: género, metodología de investigación social, economía, sociología política, sociología urbana, sociología del trabajo, sociología de la familia, estudios culturales, estudios institucionales y desarrollo conceptual; siendo género y sociología urbana los temas menos trabajados, y desarrollo conceptual el tema en el que contribuyeron la mayoría de mujeres.

Los estudios institucionales ocupan un segundo puesto en la temática más desarrollada por mujeres, seguramente gracias al proceso de institucionalización por el que pasaba la sociología en las décadas del 60, 70 y 80. Esta información es de gran utilidad para debatir acerca de las llamadas temáticas feminizadas, como lo pueden ser la sociología de la familia, género y metodologías de investigación; temas que fueron adjudicados a las mujeres en diferentes ocasiones, como sucedió en el caso mencionado anteriormente del College Junior para mujeres en la Escuela de Chicago, en este caso se desbarata el estereotipo de las temáticas feminizadas con artículos de desarrollo conceptual, a partir de las teorías clásicas de la sociología. Estos resultados demuestran el interés que tuvieron las mujeres por contribuir a la construcción de conocimiento sociológico y disciplinario; y este escenario hace aún más valiosas las contribuciones de las mujeres a la sociología colombiana.

\section{ConClusiones}

La contribución de las mujeres en las revistas de sociología colombianas entre el año 1959 y 2000, fue poco representativa en un porcentaje cuantitativo, pero de valor conceptual, entendiendo en contexto histórico, social y cultural del país en cuanto a educación femenina y representación general de las mujeres en la ciencia, es posible afirmar que las mujeres sobrepasaron barreras sociales inmersas en las dinámicas de la comunidad académica de la sociología en el país; puesto que estuvieron presentes en los procesos de institucionalización de la disciplina y participaron de forma constante en el desarrollo de esta. 
Lo expuesto en este estudio ha dejado abiertas varias reflexiones relacionadas con la participación y contribución de las mujeres en la sociología global y nacional; por ahora, puede afirmarse que hay un largo camino que recorrer para alcanzar el merecido reconocimiento a las mujeres, quienes atravesando dificultades sociales, lograron culminar, difundir y legitimar sus investigaciones en artículos publicados en revistas de sociología colombianas. Sin embargo, es preocupante el poco interés institucional y disciplinar por reconocer el papel de las mujeres en la construcción de la sociología a nivel local y global, evidenciado en el número de estudios de este talante.

Uno de los resultados más pertinentes son los temas que trabajaron las mujeres en las revistas de sociología, contrario al estigma de los temas feminizados (familia, salud, infancia, cultura) se demostró que las sociólogas colombianas se enfocaron en diversos temas, entre los más estudiados economía, sociología del trabajo, desarrollo investigativo institucional y desarrollo conceptual.

Las mujeres que iniciaron este proceso junto con Orlando Fals Borda y Camilo Torres entre 1959 y 1979 fueron Virginia Gutierrez de Pineda y Anita Weiss, siendo la primera muy reconocida en el país por sus aportes a los estudios antropológicos, pero poco reconocida por sus aportes a la sociología; y la segunda no es reconocida en el ámbito académico, aunque sus publicaciones fueron de gran inspiración en estudios acerca de la sociología del trabajo. Luego, entre 1979 y 1989, existió un aumento en el número de mujeres que aportaron a través de sus artículos al desarrollo de la sociología, en parte por el aumento de estudiantes mujeres en las instituciones de educación superior, cambios en la dinámica social en las universidades y por el aumento de las mujeres en la planta docente, en esta fase se resaltan mujeres como Luz Teresa Gómez de Mantilla, Ana Dolores Medina de Ruiz y Luz Gabriela Arango. Finalmente, en lo que se podría llamar una tercera fase del proceso de inserción en la década de los 90, se evidencia una consolidación más representativa del proceso de inserción de las mujeres a la disciplina, aumentando su participación de forma constante en las publicaciones seriadas institucionales. 


\section{ReferenCIAS}

Bea, L. (2016). Género y ciencia avanzada: análisis bibliométrico de la revista Nature desde una perspectiva de género. (Trabajo de grado Psicología). Universidad Jaime I.

Daza, S., y Pérez, T. (2008). Contando mujeres, una reflexión sobre los indicadores de género y ciencia en Colombia. Revista Antropología Social, (10), 29-51. Recuperado de http:// virajes.ucaldas.edu.co/downloads/virajes10_2.pdf

García, M. (2017). Inserción de la mujer en la sociología 1960-2000 en Bogotá D. C. (Tesis de pregrado Sociología). Universidad Santo Tomás, Bogotá. Recuperado de http://repository.usta.edu.co/bitstream/handle/11634/10205/Garc\%C3\%ADa2017. pdf? sequence $=1 \&$ isAllowed $=y$

García, S. (2010). La historia olvidada de las mujeres de la Escuela de Chicago. Revista Española de Investigaciones Sociológicas, (131), 11-41. Recuperado de http://www.reis.cis.es/ REIS/PDF/REIS_131_011277971391805.pdf

Jaraba, B., Guerrero, J., Gómez, Y., y López, W. (2011). Bibliometría e historia de las prácticas académicas locales: un esbozo a partir del caso de la psicología en Colombia. Avances en Psicología Latinoamericana, 29(2), 354-369. Bogotá, Colombia.

Licea de Arenas, J., y Santillán, E. (2002). Bibliometría, ¿para qué? Biblioteca Universitaria, 5(1), 3-10. México. Recuperado de http://dgb.unam.mx/servicios/dgb/publicdgb/bole/ fulltext/volV12002/pgs-03-10.pdf

Merton, R. (1977). La sociología de la ciencia. Alianza editorial.

Moss-Racusin, C., Dovidio, J., Brescoll, V., Graham, M., \& Handelsman, J. (2012). Science faculty's subtle gender biases favor male students. Proceedings of the National Academy of Sciences, 109(41), 16474-16479; DOI: 10.1073/pnas.1211286109.

Páez, G. (1977). Presentación. Cuadernos de Sociología, 1(1). Bogotá: Universidad Santo Tomás. Facultad de Sociología. 
Restrepo, G. (1979). Presentación. Revista Colombiana de Sociología, 1(1). Bogotá: Universidad Nacional de Colombia. Departamento de Sociología. Recuperado de https:// revistas.unal.edu.co/index.php/recs/article/view/11031/11703

Rossiter, M. (1993). The Matthew Matilda Effect in Science. Social Studies of Science, 23(2). Retrieved from https://www.jstor.org/stable/285482?seq=1\#page_scan_tab_contents

Suárez, L. (2017). Una breve historia de las revistas científicas en Colombia o la maldición de ser editor. Universitas Humanistica, (83), 9-15. Bogotá, Colombia.

Urbizagástegui, R., y Restrepo, C. (s.f.). El desarrollo de la bibliometría y los indicadores de ciencia y tecnología en Colombia.

Varón, R. (1979). Publicaciones del Departamento de Sociología de la Universidad Nacional: 1959-1979. Revista Colombiana de Sociología, 1(1), 151-155. Recuperado de https://revistas.unal.edu.co/index.php/recs/article/view/11044/11713 